\title{
Expression of pain among Mi'kmaq children in one Atlantic Canadian community: a qualitative study
}

\author{
Margot Latimer RN PhD, G. Allen Finley MD, Sharon Rudderham BA, Stephanie Inglis PhD, \\ Julie Francis BScN, Shelley Young BSc, Daphne Hutt-MacLeod MA
}

\section{Abstract}

Background: First Nation children have the highest rates of pain-related conditions among Canadian children, yet there is little research on how this population expresses its pain or how and whether the pain is successfully treated. The aim of this study was to understand how Mi'kmaq children express pain and how others interpret it.

Methods: We conducted a qualitative ethnographic study in a large Canadian Mi'kmaq community using interviews and conversation sessions. Participants included children and youth $(n=76)$, parents $(n=12)$ teachers $(n=7)$, elders $(n=6)$ and health care professionals $(n=13)$.

Results: Interpretive descriptive analysis was used and themes regarding pain expression, care seeking and pain management were identified. Pain expression included stoicism and hiding behaviour, and, when pain was discussed, it was via storytelling and descriptive language, such as similes. Participants reported feeling unheard, stereotyped and frustrated when they sought pain care. Frustration led to avoidance of seeking further care, perceptions of racism and repeat visits because of unsuccessful previous treatment. Participants voiced concerns about the utility of the numeric and faces pain scales to describe pain meaningfully. Positive encounters occurred when participants felt respected and heard.

Interpretation: Mi'kmaq children are stoic and often hide their pain. Community members feel frustrated and discriminated against when their pain is not identified, and conventional pain assessment tools may not be useful. If clinicians consider cultural context, build trust and allow for additional time to assess pain via storytelling or word descriptions as well as a family-centred approach, better pain care may occur.

boriginal people's health, development and survival may be compromised by our ability as clinicians to provide relevant culturally safe pain care (cultural safety is defined as a "framework for engagement with patients so that patients can assert power and control over their own health and well-being" ). One in 5 Canadians aged 12-44 years (3.6 million people) feels chronic pain, and the incidence is highest among Aboriginal households and those with low educational attainment. ${ }^{2}$ Although Canada ranks 3rd out of 177 countries on the Human Development Index, if only Canada's First Nations communities are considered, that ranking slips to an appalling 68th place. ${ }^{3}$ The 2012 First Nations Regional Health Survey ${ }^{4}$ showed that untreated pain profoundly interferes with a child's ability to achieve developmental milestones, mental health, academic success and productivity.

The Constitution Act, $1982^{5}$ defines Aboriginal as an inclusive term referring to First Nations, Inuit and Métis people. These 3 unique groups, who represent $4 \%$ of the Canadian population, have distinct histories, languages, cultural practices and spiritual beliefs. In a recent comprehensive review of
28 studies examining the epidemiology and management of pain among United States, Alaskan and Canadian Aboriginal peoples, only 5 included children or adolescents and only 1 took place in Canada. ${ }^{6}$ Results show that Aboriginal children have higher reported rates of dental pain, ${ }^{7}$ juvenile rheumatoid arthritis pain, ${ }^{8}$ headaches, ${ }^{9}$ injury, ${ }^{10}$ musculoskeletal and chest pain, ${ }^{11}$ yet these children are less likely to be treated for pain..$^{7-9}$ Much of what is known about pain care is derived from study in Western settings and acculturation tests of the findings are rarely provided. ${ }^{12}$ The purpose of this study was to understand how First Nation Mi'kmaq children and youth express pain and how others interpret it.

Competing interests: See end of article for competing interests.

This article has been peer reviewed.

Correspondence to: Margot Latimer, mlatimer@dal.ca

CMAJ Open 2014.DOI:10.9778/cmajo.20130086 


\section{Methods}

\section{Setting and sample}

This study involved community members of the Mi'kmaq First Nation, which is 1 of over 50 First Nations in Canada. This study took place in a large Atlantic Canadian Mi'kmaq community ${ }^{13}$, one of the 31 Mi'kmaq First Nation communities in Mi'kma'ki, the Mi'kmaq traditional territory. A convenience sample was selected using a purposive method, in which we invited children and youth from grades 1-12 and 4 adult groups (teachers, clinicians, parents and elders) to provide their perspectives. The number of Mi'kmaq community members participating was 114 .

\section{Design}

For this qualitative investigation, we used a community-based participatory action method, consisting of ethnographic techniques $^{14}$ including interviews and focus group (conversation) sessions. A collaborative relationship was established among the clinical researchers, the health directorate and members of the Mi'kmaq community. Community team members were partners in the design and implementation of the study. The protocol received approval from the IWK Health Centre's Research Ethics Board and a regional Mi'kmaq Ethics Committee at Cape Breton University.

\section{Data collection and analysis}

The conversation sessions included small groups of 4-6 children, youth, parents, teachers or health care professionals; the 5 elders were interviewed individually. Children and youth conversation sessions were organized by grade level with some grade levels being combined into one group (e.g, grades 2 and 3). A community liaison person and nurse facilitated the interviews and group sessions along with the principal investigator (M.L.) and co-investigator (S.I.).

Parents, teachers, clinicians, children and youth participated in 2 semi-structured small group conversations, and 2 individual interviews took place with each elder. Interviews and conversation sessions were held in the school, community centre and elders' homes. Sample questions are shown in Box 1. Collected data included participant demographics, conversation sessions (individual and group) and study investigators' field notes.

We conducted 10 group conversation sessions and 5 individual elder sessions twice over 8 months. The sessions with children, parents, teachers and health professionals were primarily in English; they were audiotaped, transcribed and any

\begin{tabular}{|ll|}
\hline Box 1: Sample of semi-structured interview questions \\
\hline \multicolumn{1}{|c|}{ English } & \multicolumn{1}{c|}{ Mi'kmaq } \\
\hline What does pain mean to you? & $\begin{array}{l}\text { Talite'timo'q tan wen } \\
\text { kesinukwat kisna jile'k? }\end{array}$ \\
\hline $\begin{array}{l}\text { What does pain look like? If } \\
\text { you have had an earache or }\end{array}$ & $\begin{array}{l}\text { Tan kesa'sik eksituawaqn } \\
\text { kisna kipit, kisna ketaqasin, tali } \\
\text { toothache, or muscle pain, } \\
\text { how would you describe or } \\
\text { draw it? }\end{array}$ \\
\hline
\end{tabular}

Mi'kmaq exchanges were translated into English for coding purposes. The primarily Mi'kmaq conversations held with 5 elders (not audiotaped) were captured via detailed field observation notes immediately following the sessions.

Interpretive descriptive analysis ${ }^{15}$ draws on various qualitative methods such as phenomenology, grounded theory and ethnography and assumes that the researcher will have some theoretical, clinical and pattern recognition knowledge before data collection. ${ }^{16}$ To examine the data using both a clinical and cultural lens, the principal investigator, the community liaison person and a First Nations nurse research coordinator used open coding and thematic analysis. Inter-rater reliability between the 3 coders was greater than $90 \%$ for final themes. Data themes emerged under the established categories of pain expression, seeking care and management. Themes were reviewed with the community research team and the elder advisor. Credibility of the themes was gleaned iteratively from transcribed data and the field notes and confirmed with the participants at the second conversation sessions and elder interviews.

Achieving data saturation is not an expected process or outcome in the interpretive descriptive method; however, various verification and rigorous techniques, such as concurrent data collection and analysis, constant comparative analysis and iterative analysis, were used. ${ }^{16}$ As a final validation for our project, we aimed to achieve the expectations set by Thorne and colleagues, ${ }^{16}$ that a rigorous piece of research should make sense of clinical issues and consider the new understanding consistent with that of the practice logic of an expert practitioner with reflective critical reasoning and extensive pattern recognition.

\section{Results}

Of the 114 community participants in this study, 76 were children and youth: 39 in grades 1-6, 19 in grades 7-9 and 18 in grades 9-12. The 38 adults included 7 teachers; 13 health care professionals (i.e., physicians, nurses, psychologist), of whom 6 were Aboriginal; 12 parents of young children; and 6 elders. One elder chose to withdraw for undisclosed personal reasons.

\section{Pain experiences}

The experiences of pain were categorized as medical or nonmedical. Medical pain included physical aches, such as earache, dental pain, musculoskeletal pain, headache, abdominal pain, and procedure-related pain from immunizations and venipunc-

\begin{tabular}{|ll|}
\hline $\begin{array}{l}\text { Table 1: Self-reported pain experiences of Aboriginal } \\
\text { schoolchildren }(\boldsymbol{n}=\mathbf{7 6})\end{array}$ \\
\hline Pain experience & No. $(\%)$ \\
\hline Earache & $44(58)$ \\
\hline Muscle ache & $43(57)$ \\
\hline Toothache & $41(54)$ \\
\hline Missed school due to pain & $40(53)$ \\
\hline Took pain medication regularly & $53(70)$ \\
\hline
\end{tabular}


ture. Nonmedical pain was the result of injuries from falls from bunk beds, trees, bicycles (regular and dirt bikes), as well as injuries from fights, abuse, self-injury and sports. Children selfreported how often they felt common medical pain, missed school because of pain and self-medicated for pain (Table 1).

\section{Pain expression, interpretation and seeking care}

Participants from all groups most commonly reported that children and youth were stoic in their pain expression and often hid their pain. Many participants said that youth "tough it out" or are expected to "be brave," the latter especially for boys. Participants consistently reported that children and youth might not cry or verbally express their pain to others. Comments suggested that children are socialized to be stoic by their parents, grandparents, aunts and uncles, and this was consistent in the conceptualization and expression of pain across all groups (Box 2).

Adult participants commented that children and youth display non-verbal pain cues via their eyes, tone of voice, gait and general body movement, but they may avoid eye contact and shy away from questions. Children in the lowest grades were more comfortable expressing their pain, but older children (after grades 4-5) said they did not want to show their pain so as not to be perceived as weak. Many young participants $42 \%$ $(n=32)$ wanted their mother or father to speak for them and,

Box 2: Participants' comments showing children's stoic response to pain made during individual interviews and conversation sessions

P1: No, she just sat there. She just held it.

P2: I said, she don't cry for nothing. They said, oh don't worry, all kids cry. I'm like no, she don't cry for nothing ... when she's in pain she won't.

HCP1: A little girl ... I guess she was abused growing up. She hid her pain.

HCP2: One little kid I work with, his mom was really concerned because he wasn't talking ... responding at all. We sent him for hearing tests. They said the blockage in his ear was really bad. That's why he is not speaking. But he didn't feel the pain.

P3: My daughter has chronic pain in her pelvic area. She is a competitive gymnast and refused to stop. Even her doctors told her not to do it. She would go somewhere in her head when she was doing it.

P4: For my kid, when she's in pain she doesn't complain. She tries to pretend she is fine, to avoid going to the doctor. I have to really read her body language because she does not want to go to the doctors. She's so scared, so she pretends she's fine.

Y1: I just won't say anything to them, save it to myself.

HCP3: I think there is a tendency to wait too long. ... By the time they get to you, it's too late. I have people say they have been in pain off and on for two and three months.

E1: We learned to take the hurt in resi [residential] school and hide it. E'e [yeah] it hurt, we just didn't show it.

E2: Our kids grow quiet and get withdrawn when they're hurt.

Note: $\mathrm{E}=$ elder, $\mathrm{HCP}=$ health care professional, $\mathrm{P}=$ parent, $\mathrm{Y}=$ youth clinicians suggested that some youth seek their parent's permission before answering.

Some participants said that children cope with pain by swearing, shouting, becoming angry or laughing. Elders commented that they were taught by their own parents to be tough and learned as children to take the hurt inflicted in residential schools.

The notion of hurt as both a concept and a word emerged in the findings. Verbal expressions of pain consisted of the word "hurt" consistently in both English and Mi'kmaq, and, in response to our question, "What does pain mean to you?" participants would reply, "a feeling of hurt" or "it just hurts." Many derivatives of the Mi'kmaq word kesa'si (meaning "I'm hurting") were noted in the transcripts (Box 3).

Pain was often expressed by telling a story including elaborate event details that caused the hurt. Community participants said that they convey their pain via stories and our data suggest that descriptive sentences using similes are a common pain communication strategy (Box 4). One elder noted, "The more pain, the more story." They also reported having difficulty describing their pain in the words expected and connect this with health care professionals' negative responses when they seek care (Box 4).

Some participants believed health care professionals stereotype them as alcohol and drug users even before the pain assessment, and they often viewed longer wait times for care as discrimination. Negative experiences were described in relation to subsequent delays in seeking future treatment, repeat trips for care because they did not feel heard or were unable to convey their pain the first time and overwhelming feelings of frustration and even anger (Box 5). Both community and clinician participants suggested that gold-standard measures, such as numeric pain rating and faces pain scales, were not effective in conveying or interpreting pain (Box 6), and clinicians commented that it was harder to get a history from a Mi'kmaq child than a non-native child.

Most positive encounters were described as occurring at the community health centre or in palliative care. Community clinicians offered the following advice to those conducting a pain assessment: show respect, allow more time to respond to questions and listen to the patient's event story. Some clinicians greet patients in the Mi'kmaq language to build trust and respect, and both clinicians and participants commented that this strengthens relationships. Box 7 contains a sample of comments from clinicians about how Aboriginal patients respond when asked about pain.

Box 3: Mi'kmaq translation for derivatives of the word hurt

\begin{tabular}{|ll|}
\hline English & \multicolumn{1}{c|}{ Mi'kmaq } \\
\hline I'm hurting & Kesa'si \\
\hline It hurts & $\begin{array}{l}\text { Kesitesk } \\
\text { Kesi'k }\end{array}$ \\
\hline Sore, aching & Kejunkwun \\
\hline Ow, ouch & Yiya (among younger children) \\
\hline
\end{tabular}




\section{Pain management}

Self-management was the most common strategy described by community members for treating their pain, and it included both pharmacologic and nonpharmacologic techniques. Young participants said that they regularly took pain medication $(n=53[70 \%])$ although some were unclear about proper doses. The most common nonpharmacologic selfmanagement strategies included distraction (video games and television), applying heat or cold, humour or traditional practices such as smudging (Box 8). (Smudging involves burning sweet grass or sage to clear the air of negativity.) Self-management was common and seemed to be linked with perceptions of self-reliance as well as frustration, low expectations and lack of trust in the primary care system.

\section{Interpretation}

In this study, community participants said that Mi'kmaq children are taught to be stoic when in pain. When they seek care, they show muted pain behaviour and communication mechanisms not consistent with standard assessment tools. Participants described their pain experiences using stories, and stoicism was evident as a main theme. Frustrating health care

Box 4: Ways community members described their pain and their perceptions of clinicians' responses during individual interviews and conversation sessions

Can describe pain based on cultural communication mechanisms

E3: We paint a picture.

E1: We're storytellers, describe in detail and then they don't believe us.

E4: If somebody tells you how to make a pie and gives you bits and pieces, you have to put it together yourself. I mean, you say, oh okay I see where that pie came from. So you have to give all these different symptoms. You have to.

$\mathrm{P} 4$ : Feels like someone is stabbing me from the inside.

P5: Feels like a heart attack.

\section{Cannot describe pain based on Western expectations}

E3: Many don't know the proper terminology to explain what's wrong with them.

P8: It's hard for us to communicate.

P3: Can't describe in words what we feel - it just hurts.

E2: But sometimes, you can't explain the pain, you can't.

Perceptions of clinician's response

E2: A lot of people get sent home and then they end up in [tertiary] hospital because they didn't believe them over there.

P9: They don't listen to us. They are racist.

P10: They may be listening but they are not hearing.

E1: He didn't even touch her back or try to visualize the pain. Wouldn't listen to her.

Note: $\mathrm{E}=$ elder, $\mathrm{P}=$ parent experiences, interpreted as discrimination, resulted when they attempted to seek help for their pain. Untreated pain resulted in missed school and pain self-management.

Our findings are consistent with results from other studies involving indigenous people. However, given the absence of rigorous Canadian-based evidence regarding Aboriginal people's expression of pain, we rely on work with people exposed to similar historical and cultural conditions in other countries. For example, Australian Aboriginal people display stoicism and silence when in pain in an effort to remain reserved and to be respected in their culture. ${ }^{17}$

Efforts to colonize Aboriginal people's lands and, specifically, coping mechanisms developed in response to abuse experienced during the residential school era may explain pain suppression behaviour. Elder participants in our study spoke of the residential school experience and learning to suppress their pain response. According to the Legacy of Hope Foundation, ${ }^{18}$ an estimated 180000 Aboriginal children were placed in residential schools between 1930 and 1996. Many of these children were physically, sexually, emotionally and spiritually abused, and an estimated 80000 people alive today were subjected to forced assimilation efforts. The lingering impact of the abuse in these schools, coupled with an oral, storytelling culture, may influence culturally learned pain expression and health-seeking behaviour.

In studies involving Native Americans, participants often reported pain only when it was severe and used vague descriptions, such as "ache."19,20 In our study, although the researchers asked about "pain," the respondents replied using words related

\section{Box 5: Comments on their perceptions of health care discrimination made by community members and youth during individual interviews and conversation sessions}

P2: My daughter ... she was just sitting there quiet. She'll tell me if she's sore, but she was just holding it in. Then she said, "I want to see a doctor mom," and it took four or five hours before we saw the doctor, and we finally went in they stitched it up. And there was like 50 people that came through and were gone two hours later and they kept passing us.

$\mathrm{P} 1$ : Sometimes you have to scream or make a huge deal in order to be seen.

P2: So I went to the register person and I said if you don't see my daughter now, I'm going to call the cops. I said look at my daughter's finger. I took the bandage off and they seen the gash. And she said Oh my God, and I said yeah! I told you that first time. They took her in right away.

P7: They looked at me, do you smoke? I said nope, because I had quit smoking at that time.

E1: My family doctor told me one day, are you drinking? I told em, I never drink. I never touch the liquor myself. She never asked me again if I was drinking.

E3: There's a student up there, remember [name withheld], she fell ah and she went to the hospital and they sent her back home. And she died the next day.

Y2: At the hospital it took me 8 hours just to check my collar bone. A lot of people kept going in even though I got there before them.

Note: $\mathrm{E}=$ elder, $\mathrm{P}=$ parent, $\mathrm{Y}=$ youth . 
to "hurt." There is no word for pain in the Mi'kmaq language, but there is for the word hurt (kesa'si). Although pain is a universal concept, language and cultural factors could affect the communication and interpretation of pain and, consequently, clinician assessment. This study advances our understanding of how pain is conceptualized and expressed and is a guide to how we should expect to receive information about pain from Mi'kmaq people, including from an oral, storytelling perspective.

Historically, Aboriginal people have a strong oral history: values, beliefs and complex ideas are expressed by way of stories. ${ }^{21}$ The purpose of the storytelling is to draw listeners into the experience of the event, allowing them to come to their own conclusions concerning the degree of pain. ${ }^{22}$ Community-based clinician participants recommended connecting on a cultural level by greeting patients in Mi'kmaq and making time to listen to the pain experience.

Clinicians said children were shy, avoided eye contact (possibly out of respect) and wanted a parent or guardian to answer their pain questions. In comparison, Australian indigenous people rely on relatives to report suffering and changes in character or behaviour of other family members to clinicians. ${ }^{17}$ Evidence suggests that a lower pain level is perceived among people of races different from the person doing the assessment, ${ }^{23}$ resulting in poorer treatment. ${ }^{24}$ The Canadian Hospital Accreditation Guidelines require that a child receive a pain assessment using "validated" tools on hospital admission and in association with every painful procedure. ${ }^{25}$ Western-trained health clinicians are taught to determine first the location, intensity and quality of the pain using standard validated self-reporting tools and to observe body and behavioural cues. ${ }^{26-28}$ The notion of overt pain behaviour, such as crying and facial grimace, promoted in Western culture may not be appropriate indicators of pain in Aboriginal children and youth. Reliance on self-reporting assumes that the reporter is comfortable or has a language to describe his or

Box 6: Remarks on the use of pain scales made by health care professionals and parents during conversation sessions

HCP1: I do ask people to rate their pain, but then again I take it with a grain of salt what they give me back.

HCP2: I don't use any valid way. I think the 1 to 10 scale is ridiculous.

HCP3: I don't think there is any way to objectify. It's a subjective interpretation. The scale might work if you are in the middle of treating someone you know it was a 5, now it's a 6 . I might buy into that, but to just walk in and say my pain is a $9 / 10$, that means nothing to me.

HCP4: I see tons of people who say their pain is $9 / 10$ and they are carrying on their activities.

P2: Frig, I don't really tell them that it's a 10 , because I don't know how to compare it to if someone else is more hurt than me? I usually say uh, it's a 5 ?

P5: Because that's what they ask me what does it feel like from 1 to 10 , and isn't it what everyone's opinion on what it is from 1 to 10 ?

P7: Yeah I usually say 8 or 9 usually. It doesn't really make sense.

Note: $\mathrm{HCP}=$ health care professional, $\mathrm{P}=$ parent. her pain similar to Western-held values. Better pain assessment and treatment may occur if clinician knowledge reflected cultural experiences.

\section{Limitations}

The study was conducted in only 1 rural Mi'kmaq community, and the results may not represent the perceptions of

\section{Box 7: Comments made by health care professionals during conversation sessions on how Aboriginal patients respond when asked about their pain}

HCP1: They kind of turn away. Like for children anyways, there is a kind of shyness. They don't want anyone to be near them.

HCP2: I find they look more to their family member to discuss it.

HCP3: They defer, not just kids I find.

HCP4: If there is someone else in the room, they always defer. Young adults who can clearly tell you how they are feeling just go mute and won't answer your questions.

HCP3: I do find Native kids a little more stoic, they won't tell you anything.

HCP5: I still think you have to look for the crying, grimacing, holding a body part.

HCP1: It's like they look to ask permission too [from family members]. Before they say anything to you.

HCP6: I don't know if it is a respect thing or what it is.

HCP7: Some come right out and blurb whatever, then others don't want to say

HCP3: Compared to non-Native kids, I find it's a lot tougher to get a history of any sort from a child here.

HCP8: I bust out my Mi'kmaq and talk to them. I even try and say Now listen kid, we are going to talk. And you ask the parents to ask the child to talk. And it's almost like the parents don't want that. They want to do it.

Note: $\mathrm{HCP}=$ health care professional.

\section{Box 8: Strategies for pain self-management described by} youth during conversation sessions

Y1: It's all in your mind. Pain is always in your mind. You can get rid of it like nothing. You just think pain is not really there. You just think about something else and forget about the pain eventually. The pain will just disappear.

Y2: This is what my grandma used to do. Like let's say you felt a sharp pain on your thumb .... She will pinch you somewhere else and she will make you think about that pain instead. And then usually the pain in your hand will usually disappear.

Y3: Whenever I get a needle my dad will squeeze my hand.

Y4: Sometimes when I feel pain what my mom does to us. She makes us sage ourselves every couple hours and stuff, and usually the pain will go away faster.

Y5: My brother smudges his room every day. He said it gets away all the spirits ... all that stuff. But he said it like gets rid of all emotional pain he is feeling or anything, so he does that every night. Whenever I am hurt he will come in my room and smudge me before I go to bed.

Note: $Y=$ youth. 
other First Nations people or those who live in urban settings. The self-selected participants may have had more negative experiences when seeking treatment than those who did not participate, which may have influenced their responses. The data-gathering approach involved open-ended questions related to pain expression and experience. Most data were collected during group sessions, and participants may not have shared as much information as they would have in individual sessions. The elders' sessions were not audiotaped; thus, some content may have been missed in field notes after the sessions.

\section{Conclusion}

Aboriginal children represent the fastest growing group of Canadian children and have the highest rates of pain conditions, which likely interfere with their development and academic achievement. Therefore, there is an urgent need to improve the health encounter experience of Aboriginal children. For the Mi'kmaq children in our study, we learned that pain interferes with their school attendance. Cultural beliefs and historical events may have influenced children's stoicism, which may result in muted pain expression. When pain is expressed, it is often through stories. The Mi'kmaq language has no word for "pain" but does for the more encompassing word "hurt," and this may be a better assessment term. Seeking treatment for pain has often been frustrating for community members, because they do not feel that their concerns are heard in the way they express themselves. Conventional mechanisms for pain assessment are thought to have little value in communicating and assessing pain. A culturally safe approach to pain care may include respect for oral traditions and less reliance on conventional pain scales. Additional research to examine cultural beliefs, behaviour and expression surrounding pain and the utility of established pain scales in this population is warranted.

\section{References}

1. Nguyen HT. Patient centered care: cultural safety in indigenous health. Aus Fam Physician 2008;37:990-4.

2. Canadian community bealth survey - annual component. Ottawa: Statistics Canada; 2013. Available: www23.statcan.gc.ca/imdb/p2SV.pl?Function=getSurvey $\& S D D S$ $=3226 \dot{d}$ lang $=e n d d b=i m d b \dot{\sigma} a d m=8 \dot{\sigma} d i s=2$ (accessed 2013 Mar. 10).

3. Canadian supplement to the state of the world's children 2009 -Aboriginal children's bealth: leaving no child behind. Toronto: Canadian UNICEF Committee; 2009.

4. First Nations regional health survey (RHS) 2008/10: national report on adults, youth and children living in First Nations communities. Ottawa: First Nations Information Governance Centre; 2012.

5. Constitution Act, 1982, s 35(2), Schedule B to the Canada Act 1982 (UK), 1982, c 11.

6. Jimenez N, Garroutte E, Kundu A, et al. A review of the experience, epidemiology, and management of pain among American Indian, Alaska Native, and Aboriginal Canadian peoples. 7 Pain 2011;12:511-22.

7. Leake J, Jozzy S, Uswak G. Severe dental caries, impacts and determinants among children 2-6 years of age in in Inuvik Region, Northwest Territories, Canada. 7 Can Dent Assoc 2008;74:519.

8. Mauldin J, Cameron HD, Jeanotte D, et al. Chronic arthritis in children and adolescents in two Indian health service user populations. BMC Musculoskelet Disord 2004;5:30.

9. Rhee H. Prevalence and predictors of headaches in US adolescents. Headache 2000;40:528-38.

10. First Nations regional longitudinal health survey (RHS) 2002/03: results for adults, youth and children living in First Nations communities. Ottawa: First Nations Centre; 2005. Available: http://fnigc.ca/sites/default/files/ENpdf /RHS_2002/rhs2002-03-technical_report.pdf (accessed 2013 Apr. 15).

11. Buchwald D, Goldberg J, Noonan C, et al. Relationship between post-traumatic stress disorder and pain in two American Indian tribes. Pain Med 2005;6:72-9.
12. Finley GA, Kristjánsdóttir O, Forgeron PA. Cultural influences on the assessment of children's pain. Pain Res Manag 2009;14:33-7.

13. Population projections by Aboriginal identity in Canada. The Daily. Ottawa: Statistics Canada; 2011. Cat no 91-552-X. Available: www.statcan.gc.ca/daily-quotidien /111207/dq111207a-eng.htm (accessed 2013 Dec 7).

14. Roper JM, Shapira J. Ethnography in nursing research. Thousand Oaks (CA): Sage Publications; 2000.

15. Thorne S, Kirkham SR, MacDonald-Emes J. Interpretive description: a noncategorical qualitative alternative for developing nursing knowledge. Res Nurs Health 1997;20:169-77.

16. Thorne S, Con A, McGuiness L, et al. Health care communication issues in multiple sclerosis: an interpretive description. Qual Health Res 2004;14:5-22

17. Fenwick C. Assessing pain across the cultural gap: Central Australian Indigenous peoples pain assessment. Contemp Nurse 2006;22:218-27.

18. About residential schools. Ottawa: Legacy of Hope Foundation; 2013. Available: www.legacyofhope.ca/about-residential-schools (accessed 2013 Sept. 28).

19. Elliott BA, Johnson KM, Elliott TE, et al. Enhancing cancer pain control among American Indians (ECPCAI): a study of the Ojibwe of Minnesota. 7 Cancer Educ 1999;14:28-33.

20. Kramer BJ, Harker JO, Wong AL. Arthritis beliefs and self-care in an urban American Indian population. Artbritis Rheum 2002;47:588-94.

21. Sinquin A. Our voices, our stories: First Nations, Métis and Inuit stories. Ottawa: Library and Archives Canada; 2009. Available: www.collectionscanada.gc.ca/stories 1020020-1000-e.html (accessed 2013 Apr. 9).

22. Fixico DL. The American Indian mind in a linear world: American Indian studies and traditional knowledge. New York: Routledge; 2003.

23. Drwecki BB, Moore CF, Ward S, et al. Reducing racial disparities in pain treatment: The role of empathy and perspective-taking. Pain 2011;152:1001-6.

24. Mills AM, Shofer FS, Boulis AK, et al. Racial disparity in analgesic treatment for ED patients with abdominal or back pain. Am J Emerg Med 2011;29:752-6.

25. Accreditation pain standard: making it happen! Oshawa (ON): Canadian Pain Society; 2005. Available: www.canadianpainsociety.ca/pdf/accreditation_manual.pdf (accessed 2013 Sept. 4).

26. Cohen LL, Lemanek K, Blount RL, et al. Evidence-based assessment of pediatric pain. F Pediatr Psychol 2008;33:939-55.

27. Fanciullo GJ, Cravero JP, Mudge BO, et al. Development of a new computer method to assess children's pain. Pain Med 2007;8(Suppl 3):S121-8.

28. Stevens BJ, Harrison D, Rashotte J, et al. Pain assessment and intensity in hospitalized children in Canada. 7 Pain 2012;13:857-65.

Affilations: Faculty of Health Professions, Dalhousie University (Latimer), Halifax, NS; Centre for Pediatric Pain Research (Latimer, Young), IWK Health Centre, Halifax, NS; Department of Anesthesia (Finley), Pain Management and Perioperative Medicine, Dalhousie University, Halifax, NS; Eskasoni Community Health Centre (Rudderham, Francis, Hutt-MacLeod), Eskasoni, NS; Unama'ki College (Inglis), Cape Breton University, Sydney, NS

Contributors: Margot Latimer, Sharon Rudderham and Allen Finley made a substantial contribution to the conception and design of the study. Margot Latimer, Sharon Rudderham and Allen Finley collected the data. Margot Latimer, Sharon Rudderham, Daphne Hutt-MacLeod, Julie Francis and Shelley Young analyzed and interpreted the data. Margot Latimer, Allen Finley, Sharon Rudderham, Julie Francis, and Shelley Young drafted the article. All authors revised the article critically for important intellectual content, approved the version submitted for publication and have agreed to act as guarantors of the work.

Funding: This research was funded by the Nova Scotia Health Research Foundation (NSHRF grant number 2011-7467) and the Atlantic Aboriginal Health Research Program.

Acknowledgements: The authors thank the children, youth and community members for sharing their perspectives. They also acknowledge the assistance of advanced practice nurse, Melissa Devine, in the preparation of the manuscript.

Competing interests: Shelley Young and Julie Francis received personal fees from Atlantic Aboriginal Health Research Program and Nova Scotia Health Research Foundation during the conduct of the study. Allen Finley and Margot Latimer received grants from Atlantic Aboriginal Health Research Program and Nova Scotia Health Research Foundation during the conduct of the study. No other competing interests declared.

Supplemental information: For reviewer comments and the original submission of this manuscript, please see www.cmajopen.ca/content/2/3 /E133/suppl/DC1 